\title{
Microsphere-based antibody assays for human parvovirus B19V, CMV and T. gondii

Yilin Wang ${ }^{1 *}$, Lea Hedman², Maria F. Perdomo1, Amal Elfaitouri ${ }^{3}$, Agnes Bölin-Wiener ${ }^{3}$, Arun Kumar ${ }^{1,4}$, Maija Lappalainen ${ }^{1,2}$, Maria Söderlund-Venermo ${ }^{1}$, Jonas Blomberg ${ }^{3}$ and Klaus Hedman ${ }^{1,2}$

\begin{abstract}
Background: Human parvovirus B19 (B19V), cytomegalovirus (CMV) and Toxoplasma gondii (T. gondii) may cause intrauterine infections with potentially severe consequences to the fetus. Current serodiagnosis of these infections is based on detection of antibodies most often by EIA and individually for each pathogen. We developed singleplex and multiplex microsphere-based Suspension Immuno Assays (SIAs) for the simultaneous detection of IgG antibodies against B19V, CMV and T. gondii.

Methods: We tested the performances of SIAs as compared to in-house and commercial reference assays using serum samples from well-characterized cohorts.

Results: The IgG SIAs for CMV and T. gondii showed good concordance with the corresponding Vidas serodiagnostics. The B19V IgG SIA detected IgG in all samples collected $>10$ days after onset of symptoms and showed high concordance with EIAs (in-house and Biotrin). The serodiagnostics for these three pathogens performed well in multiplex format.

Conclusions: We developed singleplex and multiplex IgG SIAs for the detection of anti-B19V,-CMV and -T. gondii antibodies. The SIAs were highly sensitive and specific, and had a wide dynamic range. These components thus should be suitable for construction of a multiplex test for antibody screening during pregnancy.
\end{abstract}

Keywords: Microsphere-based suspension immuno assay, Singleplex and multiplex, B19V, CMV, T. gondii, Pregnancy

\section{Background}

Human parvovirus B19 (B19V), cytomegalovirus (CMV) and Toxoplasma gondii (T. gondii) are ubiquitous pathogens causing diverse clinical manifestations. Their primary infections during pregnancy may lead to severe sequelae. Thus, the serological status of the mother is critically important in counselling and recognition of acute infection [1-3].

The current standards in antibody detection are enzyme-immuno assay (EIA) and chemiluminescence immune assay (CLIA) performed individually for each pathogen; the cost of which has limited its widespread use particularly when related to the overall low incidence of these conditions [4]. Multiplex arrays could be a feasible alternative thanks to their high-throughput

\footnotetext{
* Correspondence: yilin.wang@helsinki.fi

${ }^{1}$ Virology, University of Helsinki, Haartmaninkatu 3, Fl-00290 Helsinki, Finland

Full list of author information is available at the end of the article
}

performance and efficiency in terms of time and cost as well as sample volume requirement.

We developed Luminex-based singleplex and multiplex microsphere Suspension Immuno Assays (SIAs) to detect IgG antibodies against these three pathogens.

We determined the performances of the SIAs in selected cohort groups (both in singleplex and multiplex format) in comparison to corresponding high-quality inhouse or commercial EIAs.

\section{Methods}

The B19V IgG SIA was set up and examined at the University of Helsinki while the CMV and T. gondii IgG SIAs were set up at the Uppsala University Hospital. The multiplex IgG SIA for the three pathogens was performed at the University of Helsinki.
C Biomed Central

(c) 2015 Wang et al. Open Access This article is distributed under the terms of the Creative Commons Attribution 4.0 International License (http://creativecommons.org/licenses/by/4.0/), which permits unrestricted use, distribution, and reproduction in any medium, provided you give appropriate credit to the original author(s) and the source, provide a link to the Creative Commons license, and indicate if changes were made. The Creative Commons Public Domain Dedication waiver (http://creativecommons.org/publicdomain/zero/1.0/) applies to the data made available in this article, unless otherwise stated. 


\section{Study populations}

B19V IgG SIA: serum samples from 80 children or adults (2 to 58 years of age, median 10 years) with symptomatic B19V infection $[1,5]$. These included single samples from 16 subjects and 171 samples from 64 subjects followed up serologically for up to 700 days after primary infection. As control group we included serum samples from 104 constitutionally healthy medical students. The seroprevalences of $\mathrm{B} 19 \mathrm{~V}$ and several emerging viruses $[6-8]$ have been previously determined with these samples.

CMV and T. gondii IgG SIAs: serum samples from 72 subjects; single sera from 64 subjects and two consecutive sera from eight subjects. These samples have been tested for IgGs against T. gondii and CMV by the corresponding Vidas enzyme linked fluorescent assays (ELFAs, bioMérieux) and in-house B19V EIA.

For multiplex IgG SIA we used a total of 80 samples as defined above in CMV and T. gondii IgG SIAs.

\section{Coupling of antigens to magnetic/non-magnetic microspheres}

The following antigens were coupled with microspheres: for B19V IgG, cloned and purified in-house recombinant VP2 virus-like particles (VLPs) [1, 5, 9]. For CMV IgG, we used purified viral lysate (strain AD 169; Advanced Biotechnology) and for T. gondii IgG, purified tachyzoite lysate (RH strain; Advanced Biotechnology). The antigens and conditions for each assay are presented in Table 1.

The B19V-magnetic microsphere coupling was performed according to manufacturer's protocol ("Sample protocol for two step carbodiimide coupling of protein to magnetic microsphere", Luminex based xMap technology). In each coupling, a total of $1.25 \times 10^{6}$ microsphere $(100 \mu \mathrm{l})$ were coated with the corresponding antigen/protein followed by blocking with $\mathrm{PBS}+50 \mathrm{mM} / \mathrm{L}$ Tris + $0.5 \mathrm{~mL} / \mathrm{L}$ Tween-20 [10]. The coupled microspheres were washed twice with StabilGuard buffer (SG, SurModics) and stored in $1 \mathrm{~mL} \mathrm{SG}$ at $4{ }^{\circ} \mathrm{C}$ in the dark. The antigen concentration was optimized by titration ranging from 200 ug to 0.8 ug per coupling.
The CMV- and T. gondii- microsphere couplings were performed using non-magnetic microspheres as described by Elfaitouri et al. [11].

\section{Internal controls}

\section{Naked microspheres}

To estimate nonspecific binding, each test sample was run together with uncoupled ("naked") microspheres stored in SG.

\section{Suspension immunoassays}

The B19V SIA was performed following the manufacturer's protocol ("Sample protocol for indirect antibody capture immunoassay"). The B19V SIA was optimized with serial serum dilutions ranging from 1:5 to 1:320 in phosphate buffered saline with $0.05 \%$ Tween-20 (PBST). The optimal serum dilution is presented in Table 1 . Naked microspheres were included as background control. In brief, $50 \mu \mathrm{l}$ of diluted sera were dispersed in each well of 96-well flat bottom plate. Then the diluted sera were incubated with B19V-coated and naked microspheres for 45 minutes in the dark. After 3 times washing cycles, $50 \mu \mathrm{l}$ of $2 \sim 4 \mu \mathrm{g} / \mathrm{ml}$ biotinylated protein G (Thermo Scientific) was added in each well. The plate was thoroughly mixed and incubated for 30 minutes in the dark. After 3 times washing cycles, the specific signal was developed by incubation with $2 \sim 4 \mu \mathrm{g} / \mathrm{ml}$ streptavidin conjugated phycoerythrin (R-PE, Life Technologies) in PBST. Each well was resuspended in $120 \mu \mathrm{l}$ of PBST and read on the Bio-Plex 200 instrument.

The CMV and T. gondii SIAs were performed as described [11]. They were also compared with 27 sera against the corresponding Siemens BEPIII IgG tests, with good concordance (courtesy of Bo Albinson, Uppsala; data not shown).

\section{Multiplex IgG SIA}

We tested the B19V, CMV and T. gondii IgG SIAs in the multiplex format using 80 sera with known IgG reactivity, and in the same assay conditions as specified for the B19V IgG SIA.

Table 1 Antigens and conditions used in each assay

\begin{tabular}{|c|c|c|c|c|c|}
\hline SIAs & Antigen (Ag) & Ag amount(/coupling) & Serumdilution & Cutoffcriterion & Cutoff \\
\hline \multirow[t]{2}{*}{ B19V VP2 lgG } & Recombinant VP2 & $50 \mu \mathrm{g}$ & $1: 20$ & Mean + 4 SD & Negative $<453 \mathrm{MFI}^{\mathrm{a}}$ \\
\hline & & & & Mean + 5 SD & Positive $>532 \mathrm{MFl}^{\mathrm{a}}$ \\
\hline \multirow[t]{2}{*}{ CMV IgG } & Purified virion lysate & $20 \mu \mathrm{g}$ & $1: 20$ & Mean + 4 SD & Negative $<695 \mathrm{MFI}^{\mathrm{a}}$ \\
\hline & & & & Mean +5 SD & Positive $>834 \mathrm{MFI}^{\mathrm{a}}$ \\
\hline \multirow[t]{2}{*}{ T. gondii lgG } & Purified lysate & $50 \mu \mathrm{g}$ & $1: 20$ & Mean + 4 SD & Negative $<234 \mathrm{MFI}^{\mathrm{a}}$ \\
\hline & & & & & Positive $>234 \mathrm{MFI}^{\mathrm{a}}$ \\
\hline
\end{tabular}




\section{Reproducibility}

In multiplex and singleplex SIAs, the intra-assay variability was calculated with 8 replicates in the same run, and the inter-assay variability with 6 distinct runs.

\section{Cutoff value determination}

The SIAs cutoffs were calculated by the means and standard deviations (SDs) of test values. For B19V IgG SIA, the cutoff was defined with 72 sera confirmed to be B19V seronegative by both Biotrin's and in-house B19V IgG and IgM EIAs. The sera were from children with expiratory wheezing, studied earlier for human bocavirus 1 [7]. The CMV and T. gondii cut-offs were established with separate sets of 60 sera each. These samples were IgG seronegative as confirmed by the Abott assays (Table 1).

\section{Statistical analysis}

We used two-way contingency table analysis in 'VassarStats' for the calculation of kappa value, sensitivity, and specificity. Borderline values in reference assays were excluded from the calculations. The agreement between SIA and EIA was evaluated by kappa values and defined as: poor $(<0.20)$, fair $(0.21-0.40)$, moderate $(0.41-0.60)$, good $(0.61-0.80)$, and very good $(0.81-1.00)$ [12]. Pearson correlation coeffients $\left(R^{2}\right)$ were calculated by GraphPad Prism 6 to determine the correlation of results between singleplex and multiplex IgG SIAs.

\section{Cost calculation}

The net cost was calculated by the sum of all the reagents' costs (see Additional File 1).

\section{Ethical approval}

The Helsinki University Hospital Ethics Committee approved the use of all clinical samples included in this study (Dnro 553/E6/2001, $\mathbb{S} 66,13.4 .2011$ ). The control serum samples in B19V SIA study were obtained from the medical students with informed consent. All other samples in this study were taken as part of standard care and were analyzed anonymously.

\section{Results}

\section{B19V IgG SIA}

We validated the B19V IgG SIA using samples from a) symptomatic $\mathrm{B} 19 \mathrm{~V}$ patients and b) constitutionally healthy medical students.

From the symptomatic B19V individuals we tested 143 serum samples of which 16 corresponded to single sera from 16 subjects and 127 follow-up sera from 49 subjects. We found that $90 \%(129 / 143)$ of the samples were IgG positive and 4 had borderline results. All samples collected $>10$ days after onset of symptoms showed a $100 \%$ assay concordance with B19V IgG in-house EIA (Fig. 1). The four borderline samples had been collected within 6 days of onset; three of them were weakly positive in EIA (absorbance value: 0.2-0.4), and one was negative. Overall, two discrepancies were found: one, SIA+ EIA-, was collected on day 4 and the other, SIAEIA+, on day 1 after onset (Fig. 2).

In the cohort of healthy individuals, we found a seropositivity of $60 \%$ in B19V IgG SIA. This finding was in full agreement with that of EIA (Fig. 2).

Compared to in-house EIA, the B19V IgG SIA showed $98 \%$ in both sensitivity and specificity, with very good agreement (kappa coefficient $95 \%$; CI: $0.95 \sim 1$ ). The intra-assay and inter-assay variability of B19V IgG SIA were $3 \sim 8 \%$ and $1 \sim 12 \%$.

\section{CMV and T. gondii IgG SIAs}

We used samples with known reactivity against CMV and $T$. gondii in commercial assays. In concordance with

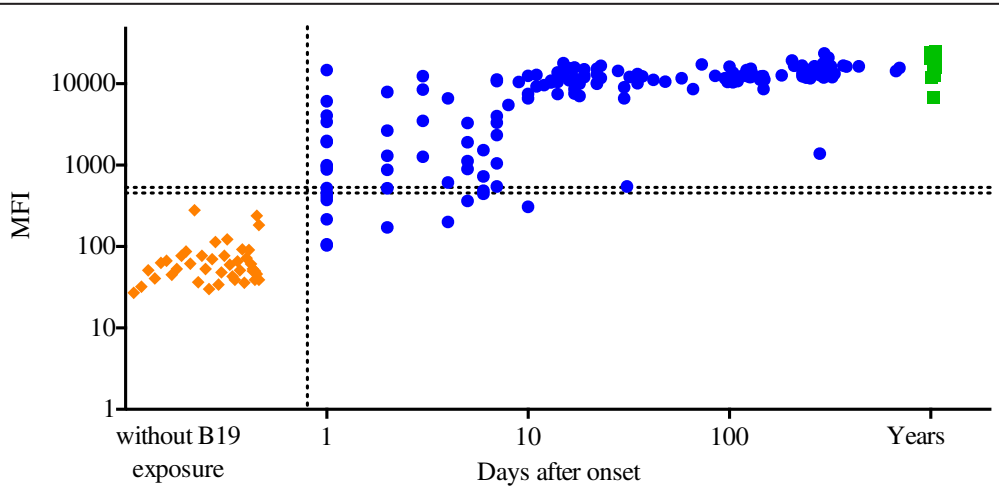

Fig. $1 \mathrm{lgG}$ response to B19V VP2. Represented are the lgG seroresponses to B19V VP2 (circle in blue) in 65 subjects with symptomatic B19V infection. The controls were asymptomatic individuals of whom 63 were lgG positive by B19V VP2 (box dot in green). Shown with diamond are the IgG seronegative healthy individuals from the same control cohort. Y-axis shows median fluorescence intensity (MFI) by SIA and $x$-axis shows days after onset. The vertical dashed line represents day 0 after onset of symptoms. The horizontal dashed lines depict the B19V IgG SIA cutoff values 


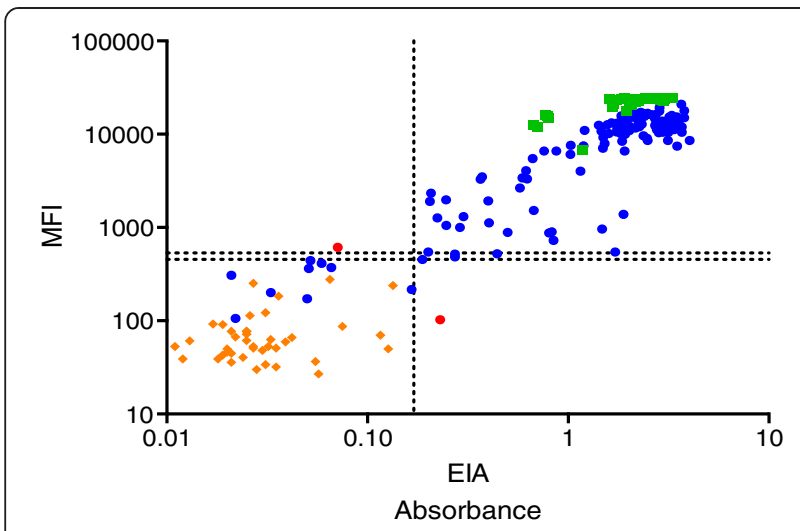

Fig. 2 Comparison of B19V IgG results by SIA and EIA. Represented is the comparison of IgG levels determined by SIA (y-axis) and EIA $(x$-axis) in sera from patients with symptomatic B19V-infection (circle in blue). Circle dots in red show two discrepancies between SIA and EIA. The controls were asymptomatic individuals of whom 63 were IgG positive by B19V VP2 (box dot in green). Shown with diamond are the IgG seronegative healthy individuals from the same control cohort. The horizontal and vertical dashed lines depict the B19V IgG SIA and EIA cutoff values, respectively

both CMV and T. gondii IgG Vidas ELFAs, we found that $75 \%(59 / 79)$ of the samples were positive in the CMV IgG SIA while $60 \%(46 / 77)$ were positive in the T. gondii IgG SIA (Fig. 3).

Compared to Vidas ELFAs, the multiplex CMV and T. gondii IgG SIAs showed $100 \%$ sensitivity and specificity; with very good agreement (kappa coefficient with $95 \%$; CI: 1).

The intra-assay and inter-assay variability of CMV IgG SIA were $3 \sim 7 \%$ and $4 \sim 9 \%$, and those of $T$. gondii IgG SIA were $7 \sim 12 \%$ and $8 \sim 15 \%$, respectively.

\section{Multiplex IgG SIA}

We tested the B19V, CMV and T. gondii IgG SIAs with 80 sera of known IgG reactivity in the multiplex format. Pearson correlation coeffients $\left(R^{2}\right)$ in Fig. 4 shows good correlation between multiplex and singleplex SIA. The B19V IgG seropositivity corresponded well with those obtained with EIA except for a single discrepancy (SIA+, EIA-). The CMV and T. gondii IgG seropositivity corresponded perfectly with those obtained with Vidas ELFA, though the assay condition as specified for the B19V IgG SIA. The good performance of the multiplex IgG SIA indicated no interference among the three IgG SIAs (Fig. 4).

The intra-assay and inter-assay variability of multiplex B19V IgG SIA were $2 \sim 8 \%$ and $1 \sim 9 \%$, of multiplex CMV IgG SIA were $4 \sim 8 \%$ and $5 \sim 13 \%$, of multiplex $T$. gondii IgG SIA were $6 \sim 9 \%$ and $8 \sim 12 \%$, respectively.
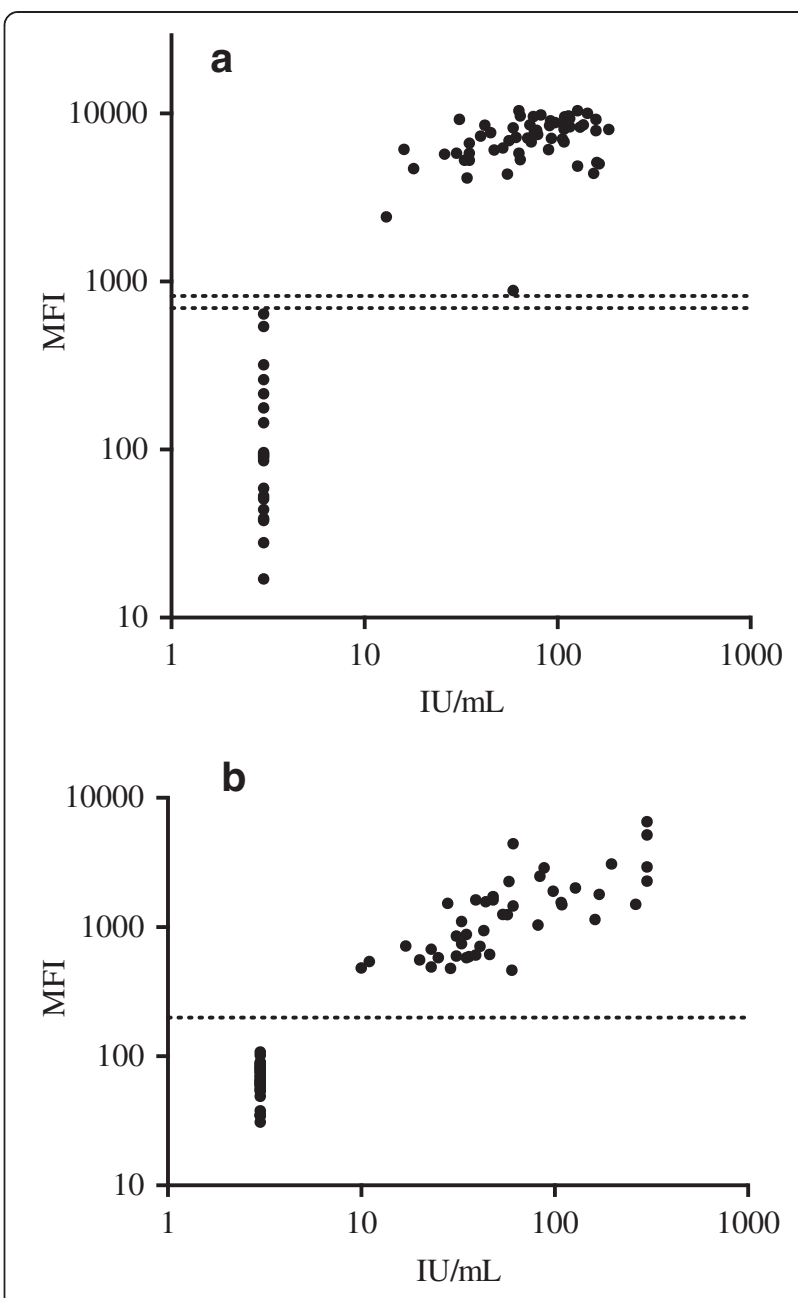

Fig. 3 Comparison of anti-CMV and anti-T. gondii lgG seroresponses by SIA and ELFA. Represented are (a) CMV IgG and (b) T. gondii lgG responses by SIAs (y-axis) or Vidas ELFA (x-axis) from 72 individuals. Horizontal dot lines indicate the cutoffs between negative and positive samples

\section{Discussion}

We established and validated singleplex and multiplex microsphere-based platforms for simultaneous detection of IgG against B19V, T. gondii and CMV. The assays were highly sensitive and specific, with no interference observable. All assays had a wide dynamic range, albeit with T. gondii lower than with B19V or CMV. The CMV and $T$. gondii IgG SIAs performed well in the multiplex format, using the same assay conditions as for the B19V IgG SIA e.g. magnetic microspheres, indicating good assay reproducibility.

The SIAs detected reliably the seroresponses against these three pathogens with an estimated net cost per sample of $0.2 €$ [13] for singleplex and $0.35 €$ for triplex, making these tools beneficial financially. Moreover, multiplex is superior to singleplex in terms of time, 


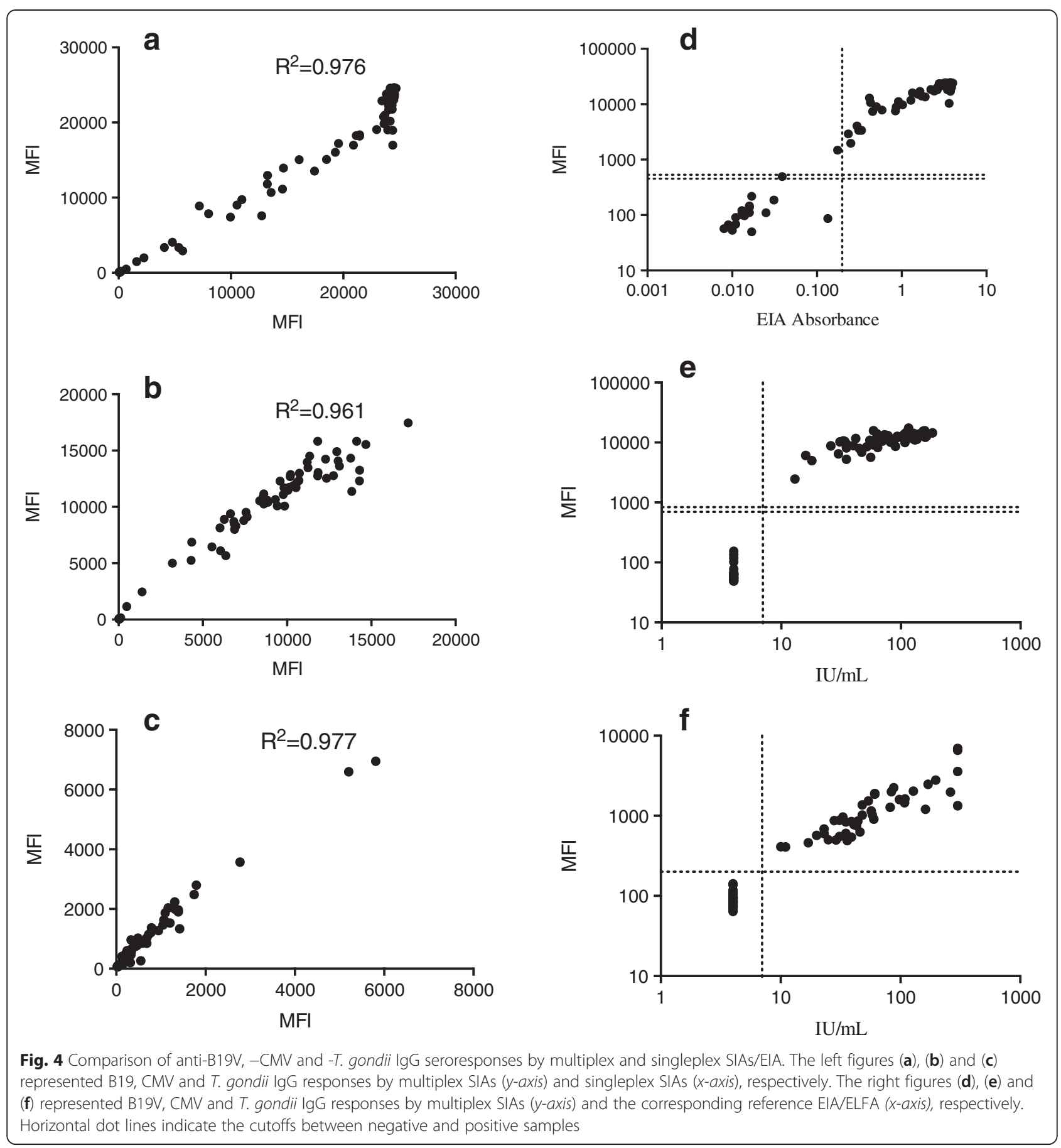

sample volume, amount of reagents and hands-on time. The multiplex- Luminex technology is flexible, and other assays maybe included in the present panel.

It also allows the possibility of including internal controls to individual samples thus providing a measure of the reliability of each readout.

Our SIA results were highly concordant with those of other immunoassays of various types (in-house EIA,
Biotrin's EIA, Vidas ELFA). The few discrepancies noted, occurred mostly in samples from early infection, possibly due to low antibody affinity. Other explanations may lay in the intrinsic differences between SIA and EIA-based reference assays [14, 15]: such as (i) fluorescence vs. colorimetric substrate detection, (ii) antigen coupling covalently onto magnetic microspheres vs. via adsorption onto streptavidin-coated plates in our in house EIAs, 
(iii) antibody detection with protein $G$ as opposed to anti-human IgG.

Of note, during the development of these assays, we found that post-coat blocking of the microsphere with bovine serum albumin (BSA) yielded inappropriately high background in some individuals (our unpublished data). However, replacement of BSA with Tris significantly improved the signal-to-noise ratios [10].

In this study, we used well-characterised cohorts of sera and controls in our singleplex and multiplex SIAs and we show good reproducibility in comparison to reference assays. However, as the test parameters were calculated based on a limited number of samples, its performance should be further validated with other clinical cohorts. Moreover, other assays such as IgG-avidity and IgM may aid in the diagnosis of acute infection and are currently under development in our lab.

\section{Conclusion}

We showed that IgG antibodies against B19V, T. gondii and CMV are successfully detected using the microspherebased suspension assay both in single and multiplex formats, making it a powerful microbiological tool.

\section{Additional file}

Additional file 1: Cost calculation. (DOCX $82 \mathrm{~kb}$ )

\begin{abstract}
Abbreviations
B19V: Human parvovirus B19; BSA: Bovine serum albumin;

CMV: Cytomegalovirus; EIA: Enzyme immunoassay; ELFA: Enzyme linked fluorescent assay; MFI: Median fluorescence intensity; PBST: Phosphate buffered saline with $0.05 \%$ Tween-20; SG: StabilGuard; SIA: Suspension immuno assay; T. gondii: Toxoplasma gondii; VLP: Virus like particle.
\end{abstract}

\section{Competing interests}

The authors declare that they have no competing interests.

\section{Authors' contributions}

YW, MF, MS, ML, KH contributed to conception and the study design of B19V SIA. YW carried out the experiment, acquisition and analysis of data in B19V and multiplex SIAs, and drafted the manuscript. LH carried out B19V lgG EIA. AK participated in production of $B 19 V$ recombinant virus like particle. $A E, A B$ and JB contributed to conception, the study design and analysis of data of CMV and T. gondii SIAs. AE and AB carried out the experiment of CMV and T. gondii SIAs. MF, MS, JB, KH helped to revise the manuscript. All authors read and approved the final manuscript.

\section{Acknowledgement}

We would like to thank the foundations at Helsinki, Helsinki University Hospital Research \& Education and Research \& Development Funds, CIMO, Instrumentarium Research Fund, The Academy of Finland, The Sigrid Jusélius Foundation and The Medical Society of Finland (FLS); and at Uppsala, ME Research UK (MERUK) and Olle Engkvist Foundation that gave financial support to this study.

\footnotetext{
Author details

${ }^{1}$ Virology, University of Helsinki, Haartmaninkatu 3, Fl-00290 Helsinki, Finland. ${ }^{2}$ Helsinki University Hospital, Helsinki, Finland. ${ }^{3}$ Section of Clinical Virology, Department of Medical Sciences, Uppsala University and Uppsala University Hospital, Uppsala, Sweden. ${ }^{4}$ Present address: T-Cell Platform, Translational Medicine, Novartis Vaccines (a GSK company), Siena, Italy.
}

Received: 16 June 2015 Accepted: 8 October 2015

Published online: 08 January 2016

\section{References}

1. Enders M, Schalasta G, Baisch C, Weidner A, Pukkila L, Kaikkonen L, et al. Human parvovirus B19 infection during pregnancy-value of modern molecular and serological diagnostics. J Clin Virol. 2006;35(4):400-6.

2. Johnson J, Anderson B. Screening, Prevention, and Treatment of Congenital Cytomegalovirus. Obstet Gynecol Clin North Am. 2014;41(4):593-9.

3. Moncada PA, Montoya JG. Toxoplasmosis in the fetus and newborn: an update on prevalence, diagnosis and treatment. Expert Rev Anti Infect Ther. 2012;10(7):815-28.

4. Khan NA, Kazzi SNJ. Yield and costs of screening growth-retarded infants for torch infections. Am J Perinat. 2000;17(3):131-5.

5. Kaikkonen L, Lankinen H, Harjunpaa I, Hokynar K, Soderlund-Venermo M, Oker-Blom C, et al. Acute-phase-specific heptapeptide epitope for diagnosis of parvovirus B19 infection. J Clin Microbiol. 1999;37(12):3952-6.

6. Lahtinen A, Kivela P, Hedman L, Kumar A, Kantele A, Lappalainen M, et al. Serodiagnosis of Primary Infections with Human Parvovirus 4. Finland Emerg Infect Dis. 2011;17(1):79-82.

7. Soderlund-Venermo M, Lahtinen A, Jartti T, Hedman L, Kemppainen K, Lehtinen $P$, et al. Clinical assessment and improved diagnosis of bocavirus-induced wheezing in children, Finland. Emerg Infect Dis. 2009;15(9):1423-30.

8. Chen T, Mattila PS, Jartti T, Ruuskanen O, Soderlund-Venermo M, Hedman K. Seroepidemiology of the newly found trichodysplasia spinulosa-associated polyomavirus. J Infect Dis. 2011;204(10):1523-6.

9. Kaikkonen L, Soderlund-Venermo M, Brunstein J, Schou O, Panum Jensen I, Rousseau S, et al. Diagnosis of human parvovirus B19 infections by detection of epitope-type-specific VP2 lgG. J Med Virol. 2001;64(3):360-5

10. Waterboer T, Sehr P, Michael KM, Franceschi S, Nieland JD, Joos TO, et al. Multiplex human papillomavirus serology based on in situ-purified glutathione s-transferase fusion proteins. Clin Chem. 2005;51(10):1845-53.

11. Elfaitouri A, Herrmann B, Bolin-Wiener A, Wang YL, Gottfries CG, Zachrisson O, et al. Epitopes of Microbial and Human Heat Shock Protein 60 and Their Recognition in Myalgic Encephalomyelitis. Plos One. 2013;8:11.

12. Altman DG. Statistics in medical journals: developments in the 1980s. Stat Med. 1991;10(12):1897-913.

13. Martins TB. Development of internal controls for the Luminex instrument as part of a multiplex seven-analyte viral respiratory antibody profile. Clin Diagn Lab Immunol. 2002;9(1):41-5.

14. Kellar KL, lannone MA. Multiplexed microsphere-based flow cytometric assays. Exp Hematol. 2002;30(11):1227-37.

15. Kellar KL, Douglass JP. Multiplexed microsphere-based flow cytometric immunoassays for human cytokines. J Immunol Methods. 2003;279(1-2):277-85.

\section{Submit your next manuscript to BioMed Central and take full advantage of:}

- Convenient online submission

- Thorough peer review

- No space constraints or color figure charges

- Immediate publication on acceptance

- Inclusion in PubMed, CAS, Scopus and Google Scholar

- Research which is freely available for redistribution 